\title{
Traitements orthodontiques accélérés par piézocision : une alternative mini-invasive aux corticotomies alvéolaires
}

\author{
Jean-David M. SEBAOUN ${ }^{1,2}{ }^{*}$, Jérôme SuRMENIAN ${ }^{1,3}$, Serge DIBART $^{1}$ \\ ${ }^{1}$ Department of Periodontology, Boston University, 100 East Newton Street, Boston MA 02118, États-Unis \\ 2115 cours Jean Jaurès, 38000 Grenoble, France \\ ${ }^{3} 23$ rue Alphonse Karr, 06000 Nice, France
}

(Reçu le 9 Mars 2011, accepté le 24 Mars 2011)

MOTS CLÉS :

Corticotomie /

Orthodontie rapide

KEYWORDS:

Corticotomy /

Rapid orthodontics
RÉSUMÉ - Avec un nombre croissant d'adultes se présentant en consultation orthodontique, la demande d'un traitement de courte durée revient de manière récurrente de la part des patients. Afin de répondre à ces attentes, plusieurs techniques ortho-chirurgicales visant à accélérer les mouvements dentaires ont été publiées mais se sont révélées relativement invasives. Nous introduisons ici une technique ortho-chirurgicale novatrice, minimalement invasive, sans lambeau muco-periosté, combinant micro-incisions, incisions corticales piézoélectriques et tunnelisation sélective permettant des greffes osseuses et gingivales. En associant plan de traitement consciencieux et compréhension des phénomènes biologiques impliqués, le métabolisme osseux alvéolaire peut ainsi être localement manipulé par cette nouvelle technique afin d'obtenir des résultats orthodontiques stables et rapides. La piézocision permet la correction rapide de malocclusions sévères sans présenter les inconvénients des approches chirurgicales extensives et traumatiques des corticotomies alvéolaires classiques.

\begin{abstract}
Accelerated orthodontic treatment with piezocision: a mini-invasive alternative to conventional corticotomies. An increasing number of adult patients are seeking orthodontic treatment and a short treatment time has become a recurring request. To meet their expectations, a number of surgical techniques have been developed to accelerate orthodontic tooth movement. However, these have been found to be quite invasive. We are introducing here a new, minimally invasive flapless procedure, combining micro incisions, piezoelectric incisions and selective tunneling that allows for hard-or soft-tissue grafting. Combined with a proper treatment planning and a good understanding of the biological events involved, this novel technique can locally manipulate alveolar bone metabolism in order to obtain rapid and stable orthodontic results. Piezocision allows for rapid correction of severe malocclusions without the drawbacks of traumatic conventional corticotomy procedures.
\end{abstract}

\section{Introduction}

\subsection{Corticotomie alvéolaire et traitements orthodontiques rapides}

Un nombre croissant d'adultes se présente en consultation orthodontique. Chez ces patients, la demande d'un traitement de courte durée revient de manière récurrente. Une accélération significative des

\footnotetext{
* Auteur pour correspondance : jdsebaoun@gmail.com
}

mouvements orthodontiques après corticotomie alvéolaire a été décrite à maintes reprises dans la littérature et ce dès la fin du XIX ${ }^{\mathrm{e}}$ siècle.

En 1959, Köle [9] décrit une technique combinant des incisions verticales du cortex inter-proximal à une ostéotomie sous apicale de vestibulaire en palatin. L'auteur explique les déplacements rapides ainsi obtenus par ce qu'il pense être des mouvements de « blocs osseux » plus ou moins indépendants les uns des autres. Par la suite, de nombreux auteurs publient des variantes de la technique en supprimant 


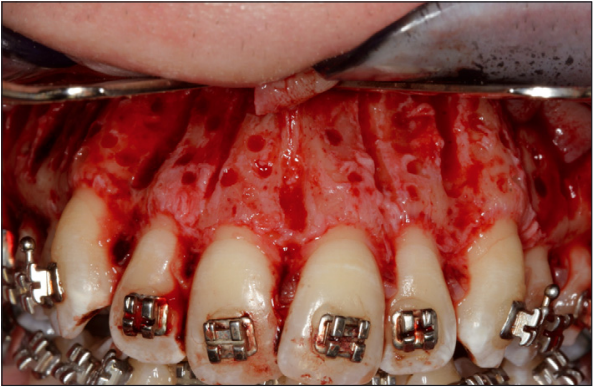

Figure 1

Corticotomie alvéolaire conventionnelle. Les incisions corticales sont réalisées au moyen d'une fraise à os après élévation de lambeaux muco-périostés en vestibulaire et palatin/ lingual.

l'ostéotomie sous-apicale et en ne réalisant que des corticotomies superficielles mais tous expliquent cependant les mouvements rapides obtenus par le concept de déplacement de blocs [1, 4, 6, 20].

En 2001, Wilcko [25] introduit une technique combinant corticotomie alvéolaire et greffe osseuse d'apposition afin de prévenir les risques de déhiscence et de fenestration tout en augmentant le champ des possibilités de corrections orthodontiques. Dans cette technique conventionnelle, des incisions corticales circonscrivant les racines sont réalisées en vestibulaire et en palatin après l'élévation de lambeaux mucopériostes (Fig. 1). La greffe est ensuite déposée en regard des dents devant être déplacées et les lambeaux sont ensuite repositionnés et suturés au niveau des papilles. Pour les auteurs [18], les déplacements rapides observés cliniquement seraient dus à un phénomène de décalcification-recalcification plutôt qu'au concept de déplacements dits de «blocs osseux ».

\subsection{Justifications biologiques}

En 1981, l'orthopédiste Frost [5] observe qu'une agression chirurgicale de l'os provoque, à proximité immédiate du site d'intervention, une augmentation du remodelage osseux ainsi qu'une diminution de la densité osseuse (Phénomène d'Accélération Régional ou RAP, Regional Acceleratory Phenomenon). Cet état ostéopénique post-chirurgical est décrit comme une condition temporaire, laissant place à une rémission complète de la densité osseuse au fur et à mesure que la cicatrisation physiologique progresse $[7,10]$.

D'autre part, de nombreux travaux ont souligné une relation entre la vitesse des mouvements dentaires et le taux de remodelage de l'os alvéolaire. Ainsi Verna [23], en induisant pharmacologiquement des taux éle- vés ou faibles du remodelage osseux démontre qu'un turnover accru est lié à un déplacement significativement plus rapide. Ces résultats sont concordants à ceux rapportés par Midgett [13] et Ashcraft [2].

Des études animales récentes viennent confirmer l'hypothèse de Wilcko selon laquelle les déplacements rapides cliniquement observés après corticotomies seraient dus au phénomène régional d'accélération de Frost et non au concept de déplacements dits de « blocs osseux».

Sur le plan histologique, nous mettions ainsi en évidence chez le rat une augmentation réversible du turnover osseux après corticotomie alvéolaire [19]. À trois semaines post-opératoire, les activités ostéoblastiques et ostéoclastiques augmentaient d'un facteur trois pour retrouver les valeurs contrôles à onze semaines. Nous notions également que ces effets se limitaient à la zone du site d'intervention.

D'autres auteurs ont évalué la qualité et la quantité des déplacements orthodontiques après corticotomie chez l'animal. Ren [17] observe ainsi des déplacements dentaires plus rapides associés à une augmentation significative du taux de remodelage osseux au niveau du site opératoire après corticotomie chez le chien Beagle. Mostafa [14] rapporte, lui, une vitesse de déplacement double après corticotomie chez le chien et attribue également cet effet à l'augmentation du remodelage osseux observé histologiquement et au phénomène d'accélération régional (RAP).

Ces conclusions concordent avec les travaux de lino [12] pour qui les déplacements accélérés sont liés aux réactions rapides au sein de l'os médullaire ainsi qu'à une moindre hyalinisation. Des observations similaires ont été publiées chez le rat après corticision (résorption et apposition accrues) et moindre hyalinisation [8].

D'autre part, en comparant les déplacements orthodontiques après corticotomie à ceux obtenus après ostéotomie, Wang [24] et Lee [11] confirment le phénomène d'accélération régionale comme l'événement responsable des mouvements rapides.

Les déplacements par distraction ostéogénique sont observés en cas d'ostéotomie et non après de simples corticotomies réfutant ainsi le concept de déplacements dits « de bloc osseux ».

\subsection{Alternatives aux corticotomies conventionnelles}

Bien qu'efficaces, les techniques de corticotomies décrites plus haut présentent des suites post-opératoires 
importantes. Le caractère agressif de ces méthodes, notamment lié à l'élévation des lambeaux mucoperiostés et à la durée de l'intervention, a suscité des réticences tant chez les patients qu'au sein de la profession.

D'autre part, l'ensemble des auteurs précédemment mentionnés réalise les incisions corticales au moyen d'une fraise à os pouvant potentiellement endommager les racines des dents avoisinantes (en cas de DDM sévère notamment dans le secteur antérieur mandibulaire).

En 2006, Park, et al. [15] introduisent la technique de corticision et suppriment les lambeaux en effectuant leurs incisions directement au travers de la gencive à l'aide d'une lame et d'un maillet chirurgical. Bien que diminuant de façon importante la durée de l'intervention, cette technique n'offre pas les bénéfices de la greffe osseuse de Wilcko.

Plus encore, au caractère très agressif de l'utilisation du maillet et de l'ostéotome au maxillaire, s'ajoute un risque de vertige paroxystique positionnel bénin [16].

En 2007, Vercellotti [22] décrit une réduction du temps de traitement orthodontique de 60 à $70 \%$ après corticotomie effectuée au moyen d'une micro-scie piézochirurgicale. De par leur petite taille et leur précision de coupe, les inserts piézoélectriques réalisent des ostéotomies précises et sans risque d'ostéonécrose [21]. L'auteur supprime le lambeau lingual en effectuant uniquement des incisions vestibulaires, mais l'élévation d'un lambeau préalable aux corticotomies reste indispensable, ne réduisant que relativement la durée chirurgicale et les suites post-opératoires.

Afin de pallier les inconvénients des techniques de corticotomies, nous introduisons une technique ortho-chirurgicale novatrice [3], minimalement invasive, sans lambeau muco-periosté combinant microincisions corticales piézoélectriques et tunnelisation sélective permettant des greffes osseuses et gingivales.

\section{Piézocizion : technique mini-invasive de corticotomie alvéolaire}

\subsection{Prise en charge parodontale initiale}

La technique de piézocision s'adressant essentiellement à des patients adultes, un bilan parodontal complet préalable à l'intervention et comprenant un relevé des lésions parodontales par sondage et un bilan radiologique rétro-alvéolaire doit impérativement être effectué.

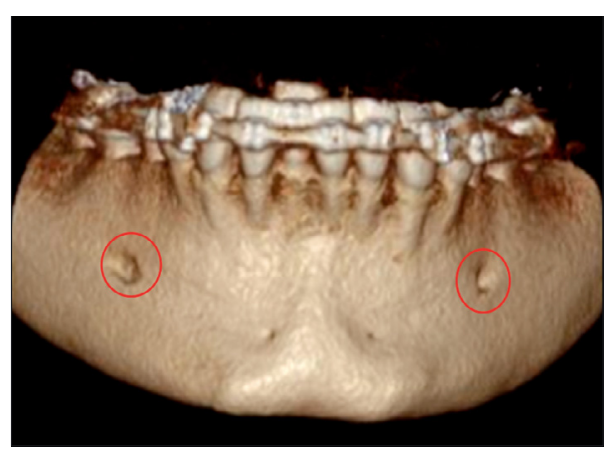

a

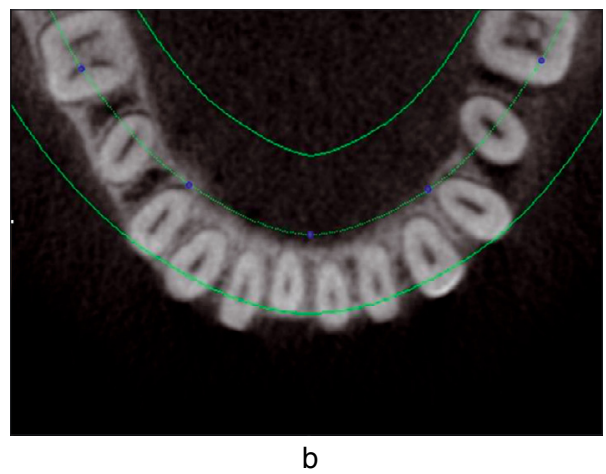

Figure 2

Imagerie 3D de type cone beam de préparation chirurgicale de l'intervention. La reconstitution pré-opératoire permettra de localiser les risques anatomiques et d'évaluer l'épaisseur de l'os cortical et les fenestrations où la greffe sera déposée.

Un détartrage systématique et au besoin un surfaçage radiculaire seront réalisés avant l'intervention chirurgicale afin d'éliminer toute inflammation tissulaire qui pourrait compromettre le bon déroulement de la cicatrisation. Toute lésion osseuse parodontale détectée devra être traitée avant d'envisager la technique de piézocision.

D'autre part, du fait de l'absence d'élévation de lambeau muco-periosté dans la technique de piézocision, nous recommandons aujourd'hui l'utilisation d'imagerie tri-dimensionnelle pré-opératoire (tomodensitométrie à faisceau conique) afin de localiser les zones de forte proximité radiculaire et les foramens mentonniers (Fig. 2). Ces images permettront également au praticien d'évaluer la quantité et la localisation des zones où un apport osseux sera indiqué.

\subsection{Intervention chirurgicale (Fig. 3)}

La chirurgie est pratiquée sous anesthésie locale, une semaine après la pose de l'appareil orthodontique. Des incisions gingivales verticales sont réalisées sous la papille inter-dentaire au moyen d'une lame 


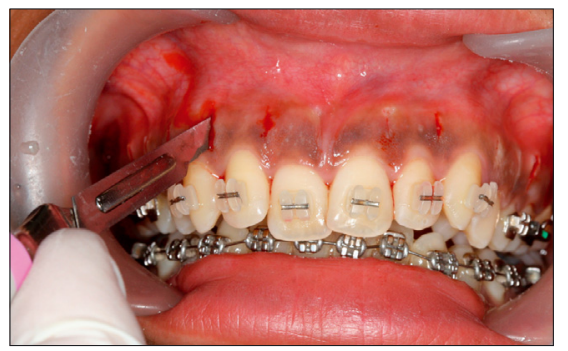

a

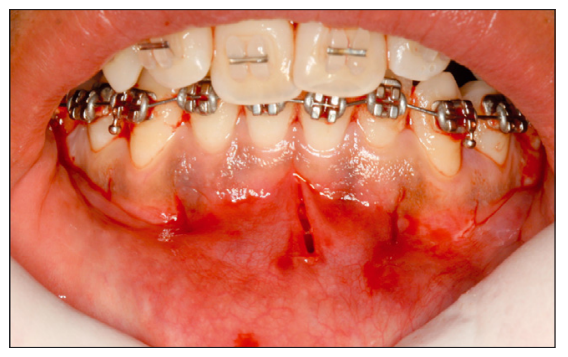

d

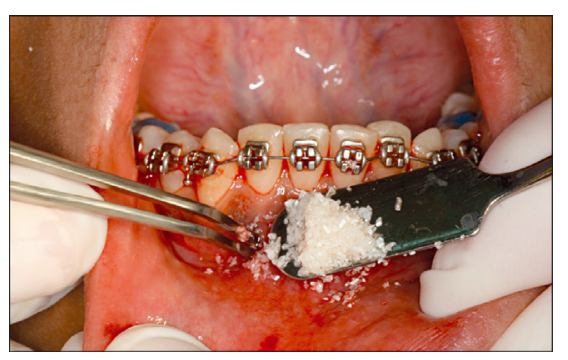

g

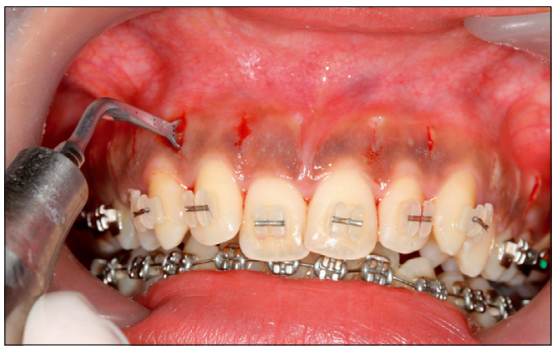

b

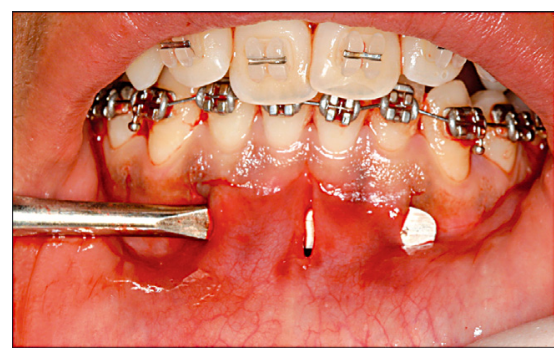

e

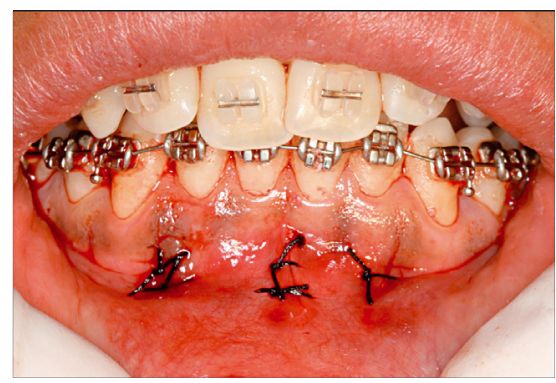

$\mathrm{h}$

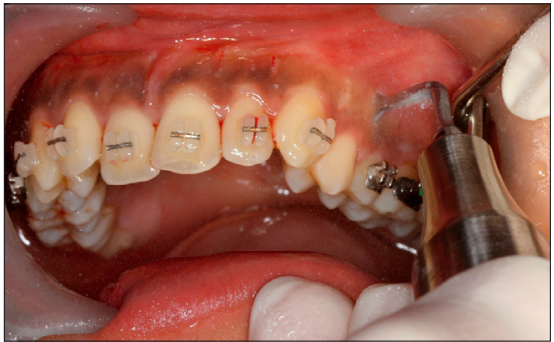

C

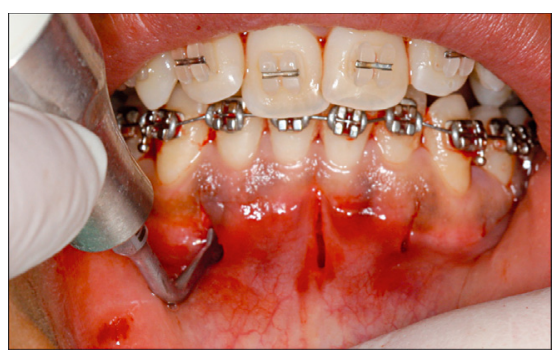

f

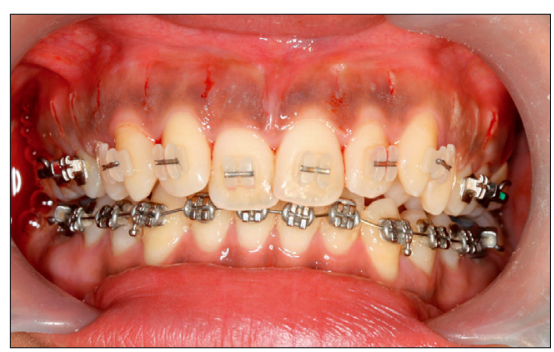

i

Figure 3

Intervention chirurgicale. (a) Les micro-incisions sont réalisées à travers la gencive attachée. (b) et (c) L'os est décortiqué à l'aide de l'insert BS-1 (Piézotome, Satelec) sur une profondeur de $3 \mathrm{~mm}$. (d) Trois incisions sont réalisées au niveau de la zone nécessitant une augmentation osseuse. (e) Un décolleur est utilisé pour créer un tunnel, afin de ménager un espace pouvant recevoir la greffe osseuse. (f) Les zones interproximales sont décortiquées. (g) Insertion de la greffe osseuse. (h) Les incisions sont refermées hermétiquement à l'aide de points simples (5.0). (i) Vue immédiate post-chirurgicale. Notez le caractère peu invasif de la chirurgie.

numéro 15 et maintenues autant que possible dans la gencive attachée. Ces incisions ne nécessitent pas d'être étendues (micro-incisions), mais doivent cependant traverser le périoste permettant ainsi à la lame d'entrer en contact avec l'os alvéolaire. Une instrumentation ultrasonique (BS1 insert, piézotome ${ }^{\mathrm{TM}}$, Satelec Acteon groupe Mérignac, France) est alors utilisée pour effectuer les traits de corticotomie au travers des micro-incisions gingivales et sur une profondeur de $3 \mathrm{~mm}$.

Notons qu'aucune suture n'est nécessaire mis à part dans les zones où une greffe osseuse est déposée.

Au niveau des zones nécessitant une augmentation osseuse, un tunnel est réalisé au moyen d'un élé- vateur inséré entre les incisions gingivales afin d'aménager un espace suffisant pour recevoir la greffe. L'allogreffe (Puros, Zimmer) est alors déposée et les incisions suturées (sutures résorbables 5-0).

Typiquement, cette greffe est réalisée en cas de DDM sévère de la zone antérieure mandibulaire. Alors que seulement trois incisions gingivales (entre les centrales et en distal des latérales) sont nécessaires à la tunnelisation, nous noterons que les incisions corticales sont réalisées entre chaque dent.

Lorsque des extractions sont indiquées, celles-ci peuvent être effectuées pendant l'intervention, le RAP obtenu par corticotomie étant limité au voisinage immédiat [19]. Il convient d'effectuer deux incisions 


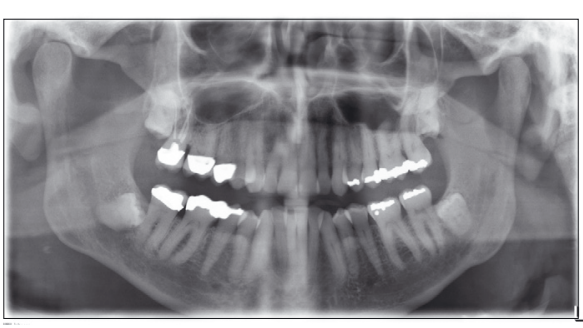

a

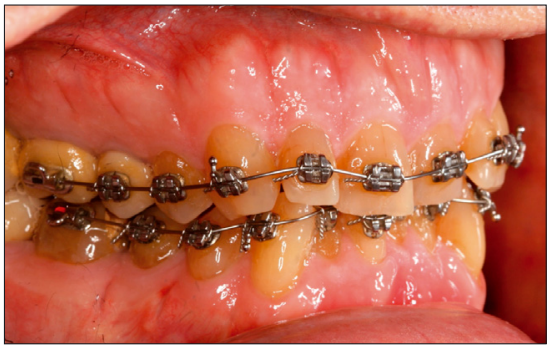

C

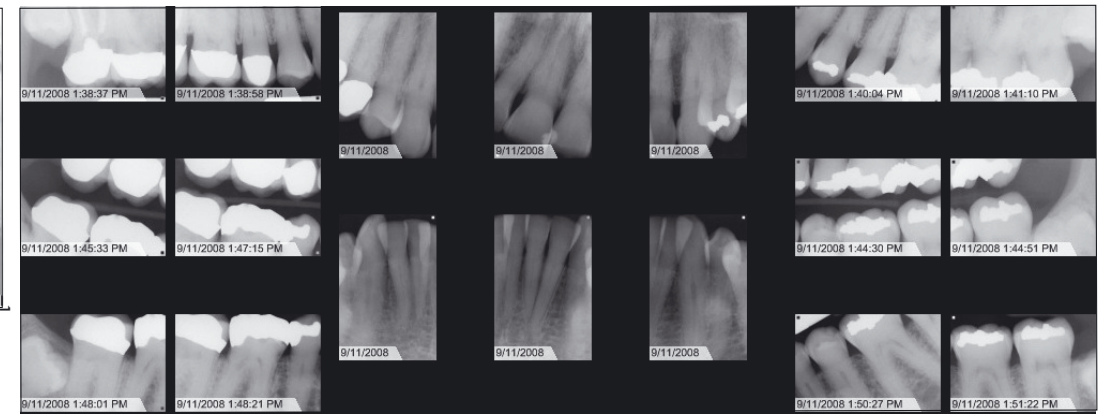

b

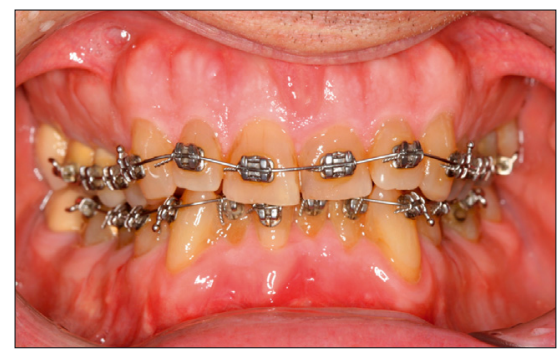

d

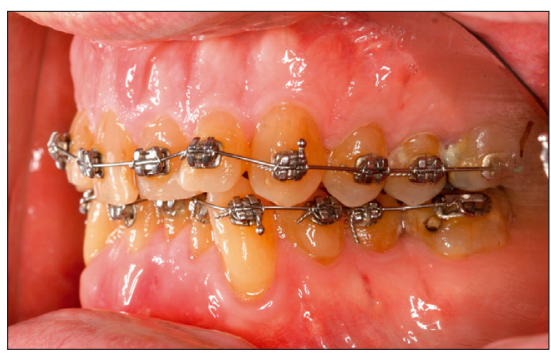

e

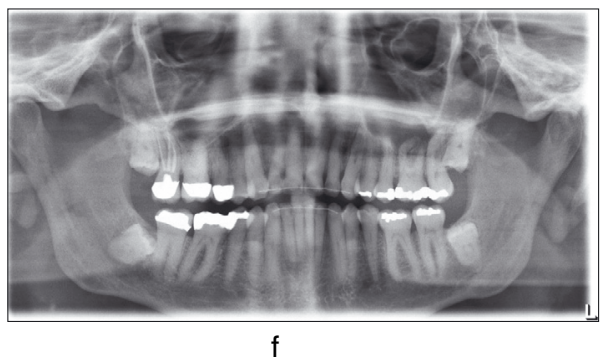

Figure 4

Suivi parodontal du patient de la figure 3. (a et b) Radiographie panoramique et status rétro-alvéolaire pré-opératoire afin d'évaluer le niveau osseux et les distances inter-radiculaires pour la planification et l'étendue des incisions corticales. (c, $d$ et e) Contrôle de cicatrisation à une semaine post-piézocison. (f) Radiographie panoramique de fin de traitement pour le contrôle du niveau osseux. Notons que les dents de sagesse du patient seront extraites ultérieurement.

corticales en regard de l'alvéole afin de faciliter la fermeture rapide de l'espace.

Soulignons que l'ensemble des incisions et la greffe, quand elle a lieu, sont effectués uniquement en vestibulaire. L'approche linguale et palatine des corticotomies conventionnelles disparaît.

Au terme de l'intervention, le patient est placé sous antibiotiques, anti-inflammatoires non stéroïdiens et bains de bouche à base de chlorhexidine. Il conviendra d'éviter de brosser les sites chirurgicaux pendant la première semaine post-opératoire afin de permettre une cicatrisation gingivale harmonieuse.

\subsection{Suivi orthodontique et parodontal}

Après l'intervention chirurgicale, les patients sont suivis à intervalles de deux semaines pour leurs ajus- tements orthodontiques. Les déplacements orthodontiques majeurs sont obtenus dans les quatre mois suivant l'opération soulignant le caractère transitoire du RAP. De notre expérience, il ressort que la majorité des cas sont traités dans un intervalle de 5 à 9 mois selon la sévérité de la malocclusion initiale.

Sur le plan parodontal, les patients sont contrôlés à une semaine post-opératoire puis tous les mois pour s'assurer du bon contrôle de plaque et renforcer au besoin les techniques d'hygiène (Fig. 4).

\section{Applications cliniques}

\subsection{Indications}

La sélection rigoureuse des cas est un facteur décisif quant à la réussite clinique de cette technique. 
Les patients doivent présenter un parodonte stable sans parodontopathie, ne pas être atteints de pathologie osseuse locale ou générale ni être soumis à un traitement de type immuno-suppressif ou biphosphonate.

La motivation et l'assiduité du patient sont essentielles en piézocision comme en corticotomie. En effet, le caractère temporaire du RAP obtenu après l'intervention nécessite une fréquence de rendez-vous plus élevée qu'en technique conventionnelle, afin de réaliser les corrections majeures dans les premiers mois du traitement.

D'un point de vue orthodontique, le candidat idéal à cette intervention présente une classe I ou classe II modérée associée à une dysharmonie dentomaxillaire modérée à sévère. Les supraclusions même importantes sont également corrigées de façon rapide après piézocision. Dans les cas extractionnels et notamment dans les cas de correction de classe II complètes, il conviendra de faire extraire les dents indiquées lors de l'intervention et d'effectuer deux à trois incisions corticales en regard de l'alvéole (Fig. 5).

D'autre part, l'intervention que nous décrivons peut être réalisée de façon segmentaire (ingression pré-prothétique, traitement d'une seule arcade...) ou être incorporée à un plan de traitement ortho-chirurgical en permettant une phase de décompensation rapide préalablement au déplacement des bases squelettiques par chirurgie orthognatique.

Enfin, l'accélération des mouvements orthodontiques observés reposant sur une modification localisée de la physiologie osseuse, la technique de piézocision peut être associée aux diverses techniques de traitements orthodontiques (vestibulaire, lingual ou Invisalign ${ }^{\circledR}$ ).

\subsection{Intérêts de la technique}

La technique que nous décrivons ici présente cliniquement des résultats similaires à ceux obtenus après corticotomie conventionnelle avec les avantages d'être plus courte à réaliser, mini-invasive et nettement moins traumatique pour le patient.

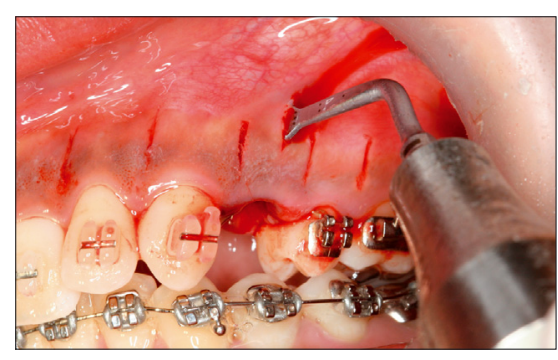

a

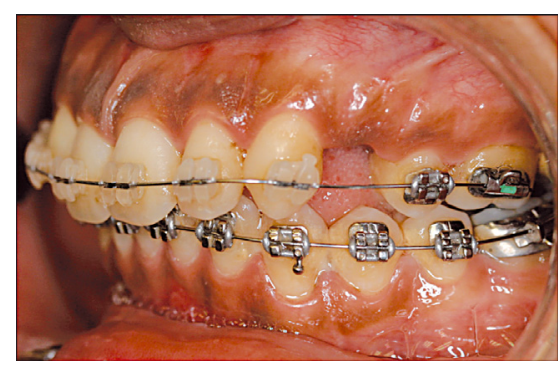

b

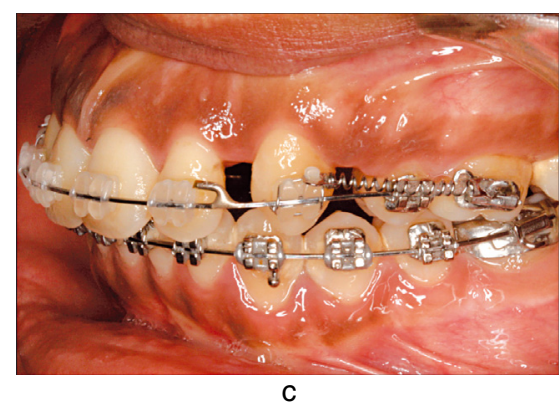

Figure 5

Correction des classes II par rétraction canine rapide après piézocision. (a) Deux à trois incisions corticales sont réalisées en regard de l'alvéole de la dent extraite et autour des dents à déplacer. (b) Avant phase de rétraction canine. (c) Quatre semaines après mise en place des ressorts de rétractions ( $300 \mathrm{~g}$ Sentalloy $\mathrm{GAC}^{\circledR}$ ).

Cliniquement, nous observons une diminution de la durée de traitement équivalente à celle obtenue par corticotomie (Fig. 6). Il ressort que la majorité des malocclusions, même sévères, sont résolues entre 5 et 9 mois. Les temps de traitement actif sont dès lors trois fois plus courts que ceux habituellement observés suite au traitement conventionnel de malocclusion similaire.

Figure 6

Patient présentant une malocclusion de classe I associée à une dysharmonie dento-maxillaire sévère (8 $\mathrm{mm})$ et une supraclusion de $50 \%$. Traitement accompli en six mois et trois semaines par piézocision maxillaire et mandibulaire de première molaire à première molaire couplée à une greffe osseuse par tunnelisation dans la zone inter-canine mandibulaire uniquement.

(a) Vue du visage de face avant traitement, (b) vue de profil en fin de traitement, (c) vue du visage de face après traitement, (d) vue de profil en fin de traitement, (e, $f$ et $g$ ) vues extra-buccales avant traitement, ( $h$, i et j) vues extra-buccales en fin de traitement. Vues occlusales : ( $k$ et $n$ ) avant traitement, (l et o) quatre mois après la piézocision, ( $m$ et $p)$ six mois et trois semaines après la piézocision. Notons que les mouvements majeurs sont accomplis dans les premiers mois faisant suite à l'intervention chirurgicale. 


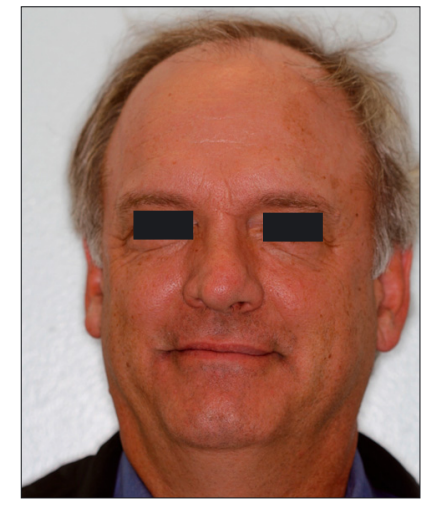

a

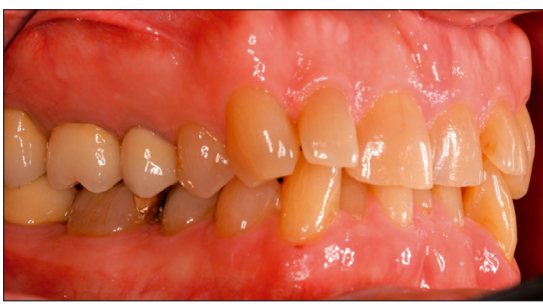

e

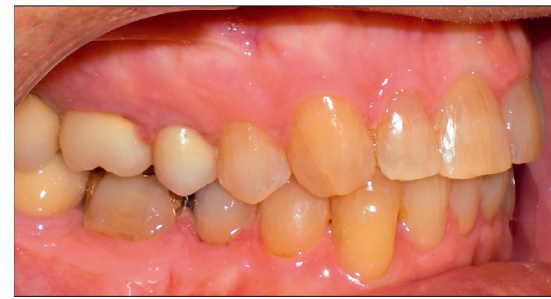

h

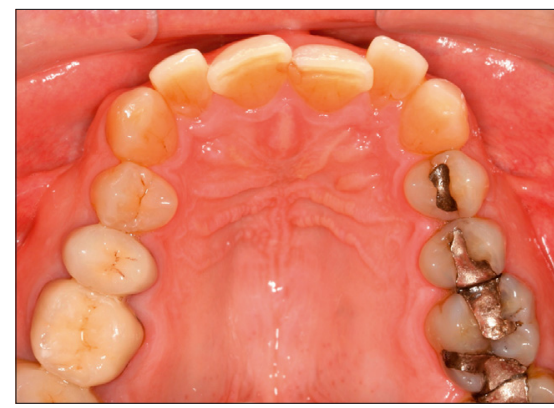

k

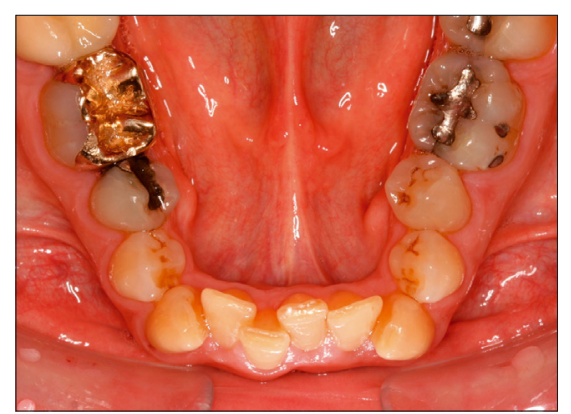

$n$

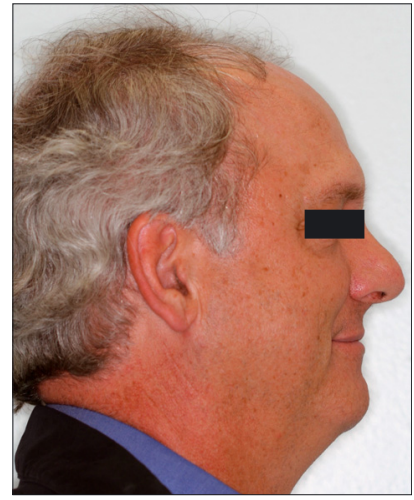

b

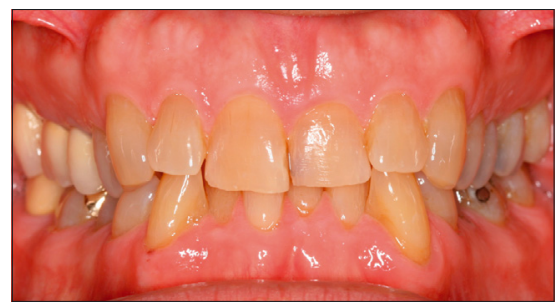

f

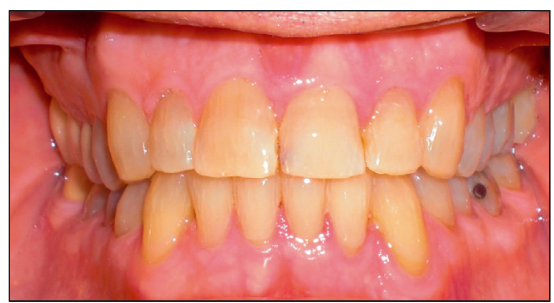

i

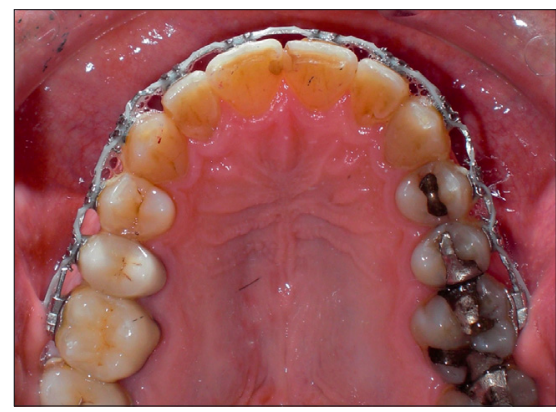

I

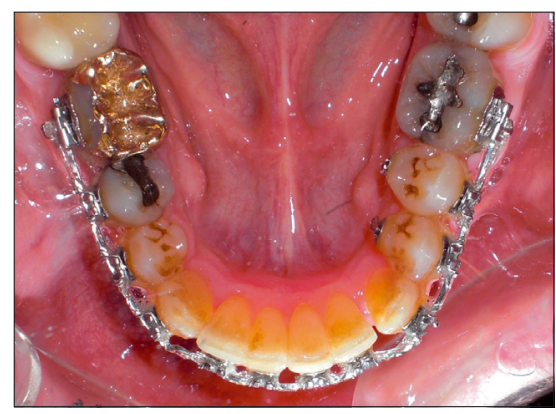

o

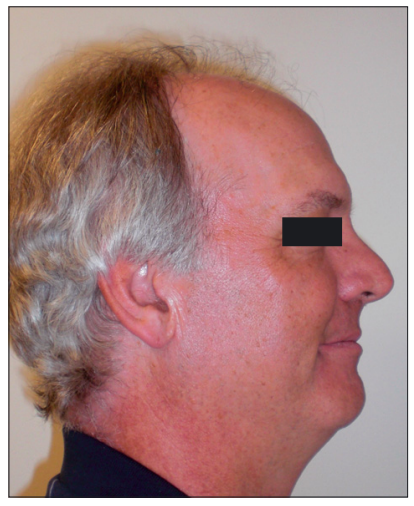

d

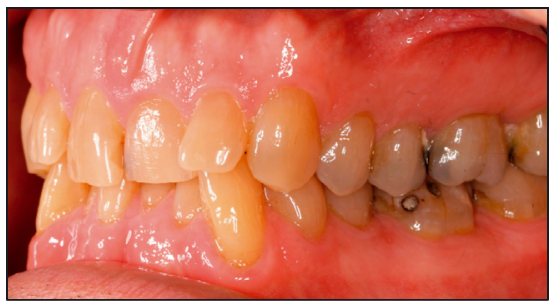

g

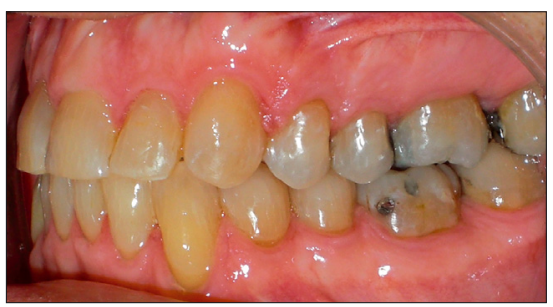

j

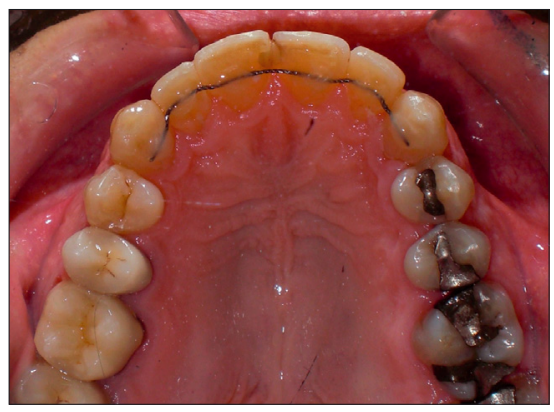

$\mathrm{m}$

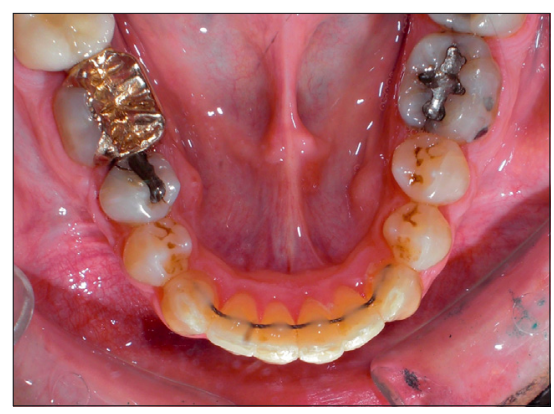

$p$

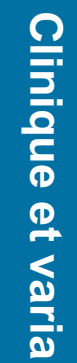


Lorsqu'une greffe osseuse est combinée à la technique de piézocision, nous notons une augmentation des possibilités de traitement notamment en permettant la correction de dysharmonies dento-maxillaires sévères sans extraction.

Sur le plan technique, 45 min à une heure suffisent généralement pour une intervention complète maxillaire et mandibulaire avec greffe contre trois à quatre heures pour les techniques classiques.

D'autre part, si les méthodes classiques pouvaient être associées à certaines complications parodontales (légère perte osseuse ou réduction de papille interdentaire), il semble que le fait de ne pas élever de lambeaux dans la technique mini-invasive écarte ces risques.

Enfin, nous noterons que les suites opératoires après piézocision sont nettement plus légères et permettent un retour à des activités normales rapidement après l'intervention. De notre expérience clinique, nous ne rapportons pas d'œdème majeur ou d'hématome de la face tel que décrit après corticotomie invasive [6]. Les douleurs post-opératoires sont généralement minimes et bien tolérées par les patients.

\subsection{Inconvénients et limites de la technique}

Du fait de l'absence d'élévation de lambeaux muco-périostés, les incisions corticales peuvent présenter un risque de lésions radiculaires notamment dans les zones de forte proximité. Un risque existe également au niveau des foramens mentonniers. Une radiographie panoramique ainsi que des vues rétroalvéolaires des zones à risque sont donc indispensables à la préparation de l'intervention. L'apport de l'imagerie tri-dimensionnelle compensera l'absence de vision directe des structures osseuses (Fig. 2).

Une précaution supplémentaire est également de rigueur quant à la localisation des incisions gingivales. Il est très important de rester au minimum à $2 \mathrm{~mm}$ de la gencive marginale afin d'éviter la formation de fentes cicatricielles.

D'autre part, en cas de pigmentations gingivales d'origine ethnique, la technique de piézocision peut créer un problème d'ordre esthétique. En effet, chaque incision est susceptible de laisser une trace qui ne se re-pigmentera pas, laissant des cicatrices et un préjudice cosmétique chez les patients présentant un découvrement gingival excessif. Il faudra donc avertir ces patients du risque potentiel de cicatrices postopératoires.

\section{Conclusion}

La piézocision est une technique ortho-chirurgicale novatrice, minimalement invasive, sans lambeau muco-periosté, combinant micro-incisions corticales piézoélectriques et tunnelisation sélective permettant des greffes osseuses et gingivales. Cette technique innovante permet la correction orthodontique de malocclusions sévères en moins d'un semestre sans présenter les inconvénients des approches chirurgicales extensives et traumatiques des corticotomies alvéolaires classiques. Elle offre un temps chirurgical réduit, des suites post-opératoires minimales, une grande tolérance chez les patients ainsi qu'un parodonte amélioré.

La piézocision se révèle comme un outil puissant dans l'arsenal de l'équipe dentaire pluri-disciplinaire chez nos patients adultes.

\section{Bibliographie}

[1] Anholm JM, Crites DA, Hoff R, Rathbun WE. Corticotomy facilitated orthodontics. Calif Dent Assoc J 1986;14:7-11.

[2] Ashcraft MB, Southard KA, Tolley EA. The effect of corticosteroid-induced osteoporosis on orthodontic tooth movement. Am J Orthod Dentofacial Orthop 1992;102:310-319.

[3] Dibart S, Sebaoun JD, Surmenian J. Piezocision: a minimally invasive, periodontally accelerated orthodontic tooth movement procedure. Compend Contin Educ Dent 2009;30:342-344, 346, 348-350.

[4] Duker J. Experimental animal research into segmental alveolar movement after corticotomy. J Maxillofac Surg 1975;3:81-84.

[5] Frost HM. The Regional accelerated phenomenon. Orthop Clin N Am 1981;12:725-726.

[6] Gantes B, Rathbun E, Anholm M. Effects on the periodontium following corticotomy-facilitated orthodontics. J Periodontol 1990;61:234-238.

[7] Henrikson P-A. Periodontal disease and calcium deficiency: An experimental study in the dog. Acta Odontol Scand 1968;26:suppl 50:1-132.

[8] Kim SJ, Park YG, Kang SG. Effects of corticision on paradental remodeling in orthodontic tooth movement. Angle Orthod 2009;79:284-291.

[9] Köle H. Surgical operations on the alveolar ridge to correct occlusal abnormalities. J Oral Surg 1959;12:515-529.

[10] Krook L, Whalen JP, Lesser GV, Berens DL. Experimental studies on osteoporosis. Methods Achiev Exp Pathol 1975; 7:72-108.

[11] Lee W, Karapetyan G, Moats R, Yamashita DD, Moon HB, Ferguson DJ, Yen S. Corticotomy/osteotomy-assisted tooth movement microCTs differ. J Dent Res 2008;87(9):861867. 
[12] Iino S, Sakoda S, Ito G, Nishimori T, Ikeda T, Miyawaki S. Acceleration of orthodontic tooth movement by alveolar corticotomy in the dog. Am J Orthod Dentofacial Orthop 2007;131:448.e1-8.

[13] Midgett RJ, Shaye R, Fruge JF Jr. The effect of altered bone metabolism on orthodontic tooth movement. Am J Orthod 1981;80:256-262.

[14] Mostafa YA, Salah Fayed MM, Mehanni S, ElBokle NN, Heider AM. Comparison of corticotomy-facilitated vs standard tooth-movement techniques in dogs with miniscrews as anchor units. Am J Orthod Dentofacial Orthop 2009; 136:570-577.

[15] Park YG, Kang SG, Kim SJ. Accelerated tooth movement by Corticision as an osseous orthodontic paradigm. Kinki Tokai Kyosei Shika Gakkai Gakujyutsu Taikai, Sokai 2006;48:6.

[16] Peñarrocha-Diago M, Rambla-Ferrer J, Perez V, PérezGarrigues H. Benign paroxysmal vertigo secondary to placement of maxillary implants using the alveolar expansion technique with osteotomes: a study of 4 cases. Int J Oral Maxillofac Implants 2008;23:129-132.

[17] Ren A, Lv T, Kang N, Zhao B, Chen Y, Bai D. Rapid orthodontic tooth movement aided by alveolar surgery in beagles. Am J Orthod Dentofacial Orthop 2007;131:160.e1-10.

[18] Sebaoun JD, Ferguson DJ, Wilcko MT, Wilcko WM. Corticotomie alvéolaire et traitements orthodontiques rapides. Orthod Fr 2007;78:217-225.
[19] Sebaoun JD, Kantarci A, Turner JW, Carvalho RS, Van Dyke TE, Ferguson DJ. Modeling of trabecular bone and lamina dura following selective alveolar decortication in rats. J Periodontol 2008;79:1679-1688.

[20] Suya H. Corticotomy in orthodontics. In: Hosl E, Baldauf A. Mechanical and biological basics in orthodontics therapy. Heidelberg, Germany: Huthig Book Verlag GmbH 1991:207-222.

[21] Vercellotti T, Nevins ML, Kim DM, Nevins DM, Wada K, Schenk RK, et al. Osseous response following respective therapy with piezosurgery. Int J Periodontics Restorative Dent 2005;25:543-549.

[22] Vercellotti T, Podesta A. Orthodontic microsurgery: a new surgically guided technique for dental movement. Int J Periodontics Restorative Dent 2007;27:325-331.

[23] Verna C, Dalstra M, Melsen B. The rate and the type of orthodontic tooth movement is influenced by bone turnover in a rat model. Eur J Orthod 2000;22:343-352.

[24] Wang L, Lee W, Lei DL, Liu YP, Yamashita DD, Yen SL. Tissue responses in corticotomy- and osteotomy-assisted tooth movements in rats: histology and immunostaining. Am J Orthod Dentofacial Orthop 2009;136:770.el-11; discussion $770-1$

[25] Wilcko WM, Wilcko MT, Bouquot JE, Ferguson DJ. Rapid Orthodontics with alveolar Reshaping: Two Case Reports of Decrowding. Int J Periodontics Restorative Dent 2001;21:9-19. 